\title{
Kinematic Analysis of a Series of Jumps In First Position In Professional Ballet Dancers
}

\author{
Jessica Smith \\ Belmont University, jessicam.smith@pop.belmont.edu \\ Kevin Robinson \\ Belmont University, kevin.robinson@belmont.edu \\ Pat Sells \\ Belmont University, pat.sells@belmont.edu
}

Follow this and additional works at: https://nsuworks.nova.edu/ijahsp

Part of the Physical Therapy Commons

\section{Recommended Citation}

Smith J, Robinson K, Sells P. Kinematic Analysis of a Series of Jumps In First Position In Professional Ballet Dancers. The Internet Journal of Allied Health Sciences and Practice. 2021 Oct 01;19(4), Article 5.

This Manuscript is brought to you for free and open access by the College of Health Care Sciences at NSUWorks. It has been accepted for inclusion in Internet Journal of Allied Health Sciences and Practice by an authorized editor of NSUWorks. For more information, please contact nsuworks@nova.edu. 


\title{
Kinematic Analysis of a Series of Jumps In First Position In Professional Ballet Dancers
}

\begin{abstract}
Purpose: Few ballet-specific studies in the literature examine kinematics during dance activities. Sports studies have identified injury risk in athletes by observing kinematics during specific activity, but this has not yet been explored for ballet. Evaluating kinematics in dancers has the potential to identify injury risk and influence treatment for prevention and rehabilitation. Therefore, the purpose of this study was to examine the landing kinematics of professional ballet dancers during a series of 16 ballet-specific jumps. Methods: This study was a multi-factor repeated measures design. Participants ( 3 males, 16 females aged 19-33 years; first or second company members) performed 16 consecutive sautés in first position to a metronome set at 120 beats per minute. Lower extremity kinematic data was captured using the Noraxon myоMOTION ${ }^{\mathrm{TM}}$ system and was analyzed at peak knee flexion of landing on jumps 1, 8, and 15 for hip abduction (HA), hip flexion (HF), and hip external rotation (HER). Analyses included: right versus left side differences (RvL), differences between jumps 1, 8, and 15, and interaction between the specific jump and RvL. Results: Statistically significant differences (SD) were found in: RvL HA ( $p=0.000,10.68$ degrees; clinically relevant) and in HF between jumps 1 and $8(p=0.000)$ and 1 and $15(p=0.006)$. There was no interaction effect of HA between jump number and RvL $(p=0.470)$ or of HER between jump number and RvL ( $p=0.599)$. There was no SD in: HF between jumps 8 and $15(p=0.245), H A$ between jumps $(p=0.062)$, RvL HF ( $p=0.190)$, RvL HF between jumps ( $p=0.972)$, HER between jumps $(p=0.366)$, or RvL HER $(p=0.562)$. Conclusions: The RvL difference in HA ( $p=0.000)$ suggests asymmetrical loading of hip joints. This difference did not change between jumps $(p=0.062)$, suggesting repeated preferential frontal plane loading. The SD in HF between jumps 1 and $8(p=0.000)$ and 1 and $15(p=0.006)$ suggests that a small series of jumps that are standard in ballet class are not consistent motions for professional dancers. Limitations include small sample size and lack of controlled warm up. Future work should examine single leg jumps or sautés following a fatigue protocol to assess joint motion more accurately.
\end{abstract}

\section{Author Bio(s)}

Jessica Smith, SPT, BA, is a student of physical therapy at Belmont University in Nashville, TN. She received her Bachelor's degree in Psychology from Illinois Wesleyan University in 2017. Her future clinical interests include performing artists and athletes.

Dr. Kevin Robinson, PT, DSc, OCS, has over 34 years of clinical experience with the management of orthopedic and sports injuries. Dr. Robinson is an Associate Editor for the International Journal of Sports Physical Therapy. He has maintained is Certification as an Orthopedic Clinical Specialists from the American Board of Physical Therapy Specialties (ABPTS) since 1995 and is also involved in his clinical practice at the Performance Therapy Institute in Franklin, TN. His clinical practice melds his love of patient care with his formal training in biomechanics and orthopedics. He routinely performs clinical 3D gait and movement analysis to develop rehab and training programs for patients and athletes.

Dr. Pat Sells, DA, MA, ACSM-CEP, received his undergraduate degree at Tennessee Technological University in Physical Education and completed his Master's degree in adaptive Physical education there as well. After a period of time in the corporate world working for the consulting firm Arthur Anderson, Dr. Sells returned to academia and completed his Doctoral studies at Middle Tennessee State University.

Dr. Sells' clinical background in exercise physiology includes both Cardiac and Pulmonary Rehabilitation and exercise testing and prescription in athletes. Dr. Sells is certified by the American College of Sports Medicine as a Clinical Exercise Specialist (CES), as an Advanced Cardiac Life Support provider and is certified as a golf fitness instructor by the Titleist Performance Institute. Dr. Sells has research interest in 
a variety of lines, including concussion in youth sports, Blood Flow Restriction, muscle oxygen saturation, movement studies in golf, dance, and plyometric landing. Dr. Sells teaches courses in Human Physiology, Cardiopulmonary Physical Therapy, Statistics, Critical Inquiry and Health and Wellness. Dr. Sells is a reviewer for the International Journal of Sports Medicine.

Prior to joining the Physical Therapy Department, Dr. Sells served as the Director of Cardiac Rehabilitation at Vanderbilt University Medical Center. His clinical experience includes metabolic testing and rehabilitation with diverse patient populations including; heart transplant recipients, congestive heart failure, COPD, emphysema, cystic fibrosis, obesity, diabetic athletes and children.

\section{Acknowledgements}

The authors would like to acknowledge Susan Underwood Physical Therapy and the Nashville Ballet for their collaboration and participation in this research. 


\title{
IIIAHSP \\ The Internet Joutnal of Allied Health Sciences and Practice
}

Dedicated to allied health professional practice and education

Vol. 19 No. 4 ISSN 1540-580X

\section{Kinematic Analysis of a Series of Jumps in First Position in Professional Ballet Dancers}

\author{
Jessica Smith \\ Kevin Robinson \\ Pat Sells \\ Belmont Univeristy \\ United States
}

\begin{abstract}
Purpose: Few ballet-specific studies in the literature examine kinematics during dance activities. Sports studies have identified injury risk in athletes by observing kinematics during specific activity, but this has not yet been explored for ballet. Evaluating kinematics in dancers has the potential to identify injury risk and influence treatment for prevention and rehabilitation. Therefore, the purpose of this study was to examine the landing kinematics of professional ballet dancers during a series of 16 ballet-specific jumps. Methods: This study was a multi-factor repeated measures design. Participants ( 3 males, 16 females aged 19-33 years; first or second company members) performed 16 consecutive sautés in first position to a metronome set at 120 beats per minute. Lower extremity kinematic data was captured using the Noraxon myOMOTION ${ }^{\top M}$ system and was analyzed at peak knee flexion of landing on jumps 1, 8, and 15 for hip abduction (HA), hip flexion (HF), and hip external rotation (HER). Analyses included: right versus left side differences (RvL), differences between jumps 1, 8 , and 15, and interaction between the specific jump and RvL. Results: Statistically significant differences (SD) were found in: RvL HA ( $p=0.000,10.68$ degrees; clinically relevant) and in HF between jumps 1 and $8(p=0.000)$ and 1 and $15(p=0.006)$. There was no interaction effect of HA between jump number and RvL $(p=0.470)$ or of HER between jump number and RvL ( $p=0.599)$. There was no SD in: HF between jumps 8 and $15(p=0.245), H A$ between jumps ( $p=0.062)$, RvL HF ( $p=0.190)$, RvL HF between jumps ( $p=0.972)$, HER between jumps ( $p=0.366)$, or RvL HER $(p=0.562)$. Conclusions: The RvL difference in HA $(p=0.000)$ suggests asymmetrical loading of hip joints. This difference did not change between jumps ( $p=0.062$ ), suggesting repeated preferential frontal plane loading. The SD in HF between jumps 1 and 8 $(p=0.000)$ and 1 and $15(p=0.006)$ suggests that a small series of jumps that are standard in ballet class are not consistent motions for professional dancers. Limitations include small sample size and lack of controlled warm up. Future work should examine single leg jumps or sautés following a fatigue protocol to assess joint motion more accurately.
\end{abstract}

Keywords: ballet, performing arts, dance, biomechanics, jumps 


\section{INTRODUCTION}

Because of the high number of injuries that occur during jumps in ballet, there is a need to evaluate dynamic joint angles in the lower extremity in order to assess how dancers land and to identify potential reasons for these injuries..$^{1-5}$ Ballet is an incredibly active art form which both demands and lacks motion studies. Motion studies help to identify why injuries are occurring; this information should help prevent future injuries so dancers can continue performing. There are currently few studies in the literature examining joint angles during ballet-specific activities. This lack of literature leads practitioners who treat dancers to reference the sports literature instead as both sports and dance require frequent jumping and pivoting activities. ${ }^{6,7}$ In the sports literature, both landing and pivoting kinematics have been shown to predispose an athlete to injuries of the lower extremity, specifically at the knee. ${ }^{6}$ A couple of studies have compared the landing mechanics of athletes to those of dancers and found that in general, dancers have better landing mechanics compared to their athletic counterparts. $6-8$ However, these studies did not address ballet-specific jumps, leaving the question of what landing mechanics look like for dancers when they are engaged in dance.

One of the most basic ballet-specific jumps is the sauté. This term is simply translated to "jump," and is commonly used as the first jump to warm-up when transitioning in the center to the jumping section of ballet class. ${ }^{9} \mathrm{~A}$ saute in first position is a jump that begins on two feet and lands on two feet. It is important to maintain excellent form on this two-footed jump in order to safely progress to more advanced jumps later in ballet class. This study chose to examine form during a sauté in an attempt to contribute to a foundation in the literature of dancers' kinematics and where they may be lacking or having excessive motion that may contribute to injury.

As mentioned, most injuries during jumps in ballet occur in the lower extremities. ${ }^{1-5}$ Currently, the most commonly examined joint in the ballet literature is the hip. 4,5 Because of the expectation in ballet for 180 degrees of lower extremity external rotation (a 90 degree contribution from each leg), it is commonly thought that dancers compensate from other lower extremity joints to attain this amount of external rotation, which has been speculated as a cause for injury. ${ }^{10}$ Though this compensation is thought to be used, dynamic joint angles in more than a single repetition of ballet-specific movements (a single demi plié, grand plié, and a single sauté) have not been assessed. ${ }^{5}$ As a result, this study chose to examine the hip in all planes of motion (frontal, saggital, and transverse) during a series of jumps in order to build a foundation of knowledge for hip movement and whether it contributes to injury.

The purpose of this paper is to examine the landing kinematics of ballet dancers from a jump to determine whether this could explain lower extremity injuries in ballet. Side to side differences will also be observed as this has been noted as a cause for injury in athletics in the past. ${ }^{11}$ The research questions that this paper sought to answer were 1) Is there a difference in hip external rotation, abduction, or flexion between dancers between jumps 1,8 , and 15 of a simple warm up combination, and 2) Is there a difference of side to side hip external rotation, abduction, or flexion angles within dancers between jumps 1,8 and 15 , or at all?

\section{METHODS}

This study was approved by the Belmont University Institutional Review Board.

\section{Participants}

Subjects included 19 professional dancers from Nashville Ballet. There were 3 male (24.67 \pm 3.51 years old) and 16 female (24.13 \pm 4.56 years old) participants. Participants ranged in age from 19-33 years old and were all members of either the first or second company. More detailed participant demographics are presented in Table 1. Data were taken during the 2019 pre-season, which fell in mid-August.

Table 1. A representation of the demographical data of participants

\begin{tabular}{|l|l|l|l|l|}
\hline Sex & & Age (years) & Weight (lb) & Height (in) \\
\hline Male & $\mathrm{N}$ & 3 & 3 & 3 \\
& Mean & 24.67 & 154.33 & 70.92 \\
& Standard Deviation & 3.51 & 12.50 & 1.66 \\
\hline Female & $\mathrm{N}$ & 16 & 16 & 16 \\
& Mean & 24.13 & 117 & 65.06 \\
& Standard Deviation & 4.56 & 9.33 & 2.39 \\
\hline Total & $\mathrm{N}$ & 19 & 19 & 19 \\
& Mean & 24.21 & 122.89 & 65.99 \\
& Standard Deviation & 4.33 & 16.90 & 3.14 \\
\hline
\end{tabular}

(C) The Internet Journal of Allied Health Sciences and Practice, 2021 


\section{Instrumentation}

Dynamic joint angles at the hip, knee, and ankle during jumps were measured via myOMOTIONTM Kinematic Analysis System, version MR3 4.12 (Scottsdale, AZ). myOMOTION'M utilizes inertial sensors that have been shown to measure joint angles during dynamic trials with a mean error of $0.5^{\circ}$, which provided accuracy for the joint angle measurements of this study. ${ }^{12}$ The motions are able to be broken down per millisecond in a frame-by-frame retrospective analysis. For retrospective analysis, the system creates avatars of a participant's skeletons in order to provide a visual of their biomechanics. Examples of these skeletons are pictured in Figure 1. Other instrumentation used for this study includes The Metronome by Soundbrenner@ application for iPhone 6 , version 1.22.0, which was used to keep a standardized tempo to which dancers could jump.
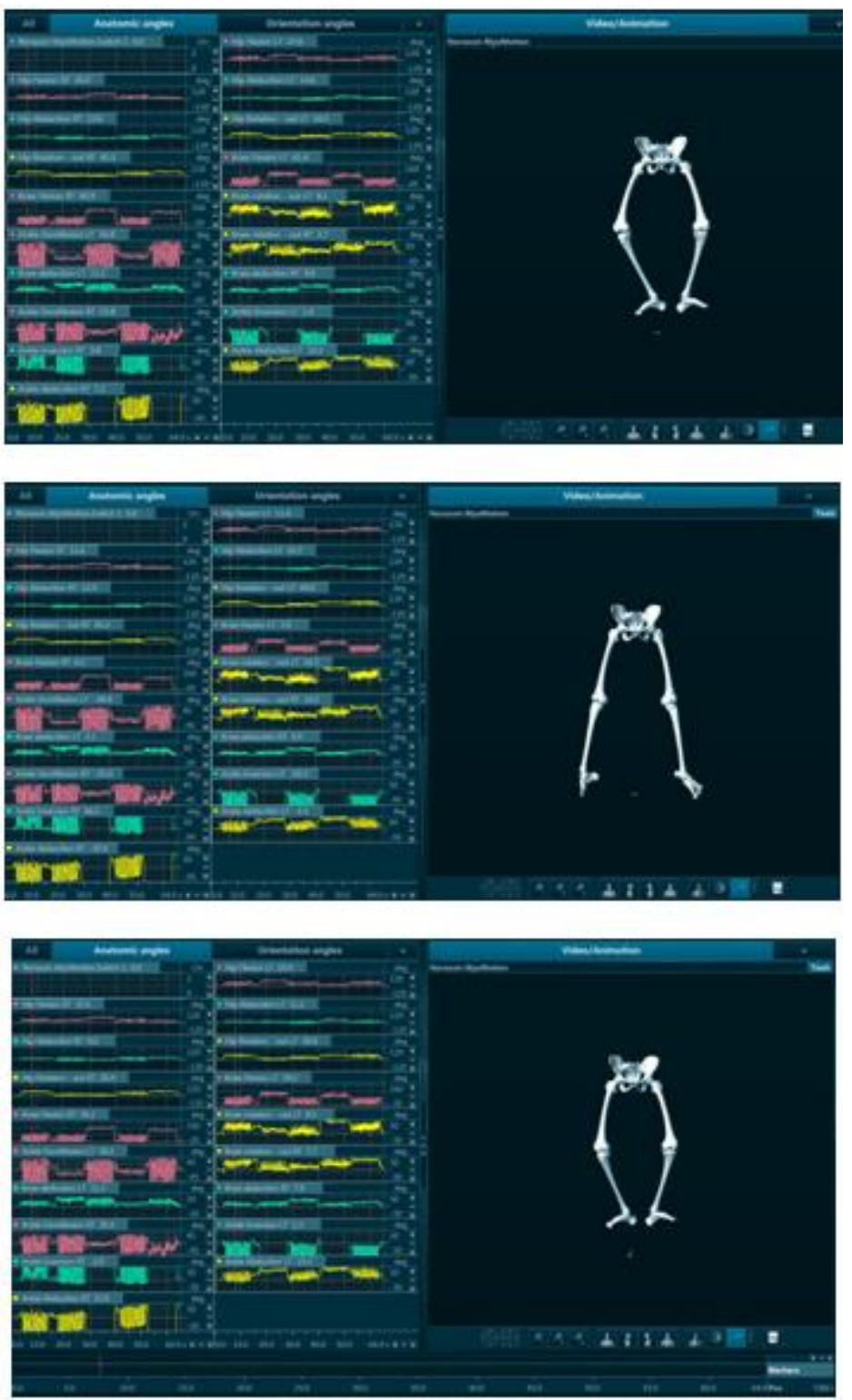

Figure 1. Avatar and real time data of a sauté in first position performed by the participants. Top: Preparation for the jump. Middle: Jump in mid-air. Bottom: Landing of the jump. 


\section{Experimental Protocol}

Inertial sensors were placed on the sacrum, lateral aspect of the distal femurs, bilateral forelegs, and the dorsum of each foot centered over the second and third metatarsals, as shown in Figure 2. Figure 2 also displays the neutral position dancers stood in to calibrate the sensors. The sensors that were used are shown in Figure 3.

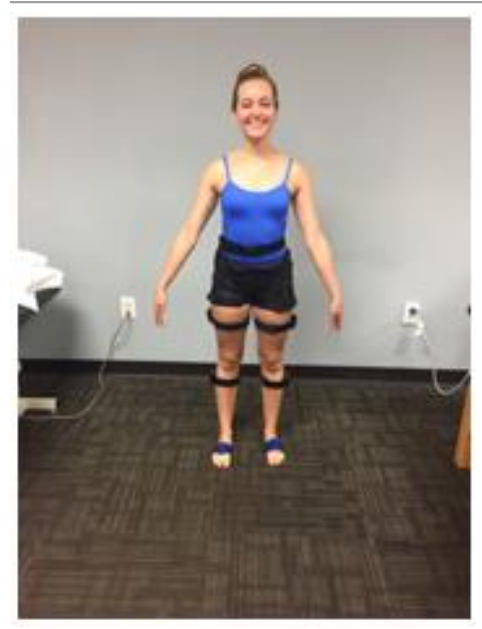

Figure 2. Setup of myOMOTION ${ }^{\top M}$ sensors in the neutral position that was assumed for calibration.

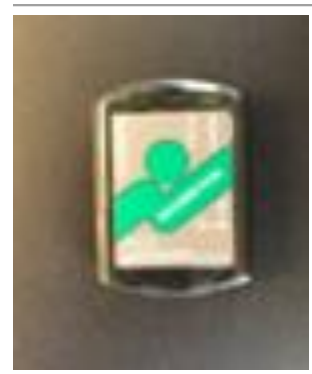

Figure 3. myoMOTIONTM sensor used for trials

Participants were asked to perform 16 consecutive sautés in first position. The breakdown of a sauté in first position is shown in Figure 4. All participants were required to follow a tempo of 120 beats per minute, ensuring consistency with each jump and mimicking what the dancer would have to do if jumping to music in class or performance. To keep this tempo, the researcher used The Metronome by Soundbrenner@ application for iPhone 6, version 1.22.0. Volume was turned up fully on the researcher's phone and each dancer was asked whether they could hear the metronome well before performing the jumps. They were also allowed as much time as they needed to mark the proper tempo before performing the jumps to ensure they kept pace. Dancers were allowed one trial of the 16 jumps, after which the sensors were removed. Prior to participation in the study, participants were allowed whatever warm up they needed. This varied, with some participants giving themselves a full ballet class, others doing a cardio warm up, and some coming in with no warm up at all. All measurements took place in the same ballet studio with a raised floor covered with marley and a full length mirror at the front of the studio. All dancers stood facing the mirror for the trial as would be customary during a ballet class.

\section{Data Processing and Analysis}

IBMC SPSS@ Statistics Version 26 was utilized for data analysis. To compare right and left sides within dancers between jumps 1,8 , and 15 , a repeated measures analysis of variance was run. The independent variable was jumps and had three levels: jump 1, jump 8 , and jump 15. The dependent variables were hip abduction, hip flexion, and hip external rotation. These measurements were taken upon landing at peak knee flexion (in ballet, the deepest part of the plié) at each jump as identified on the frame by frame of each dancer from the myOMOTIONTM analysis. Examples of the screenshots that were used for analysis from the myoMOTION ${ }^{\mathrm{TM}}$ software are shown in Figure 1. Jump 15 was used for analysis as opposed to jump 16 due to a large observed increase in knee flexion on the last jump that was inconsistent with the rest of the jumps within dancers, suggesting that taking data for jump 16 would have inaccurately represented the biomechanics of normal jumps. Right sides were compared within dancers and left sides were compared within dancers. 

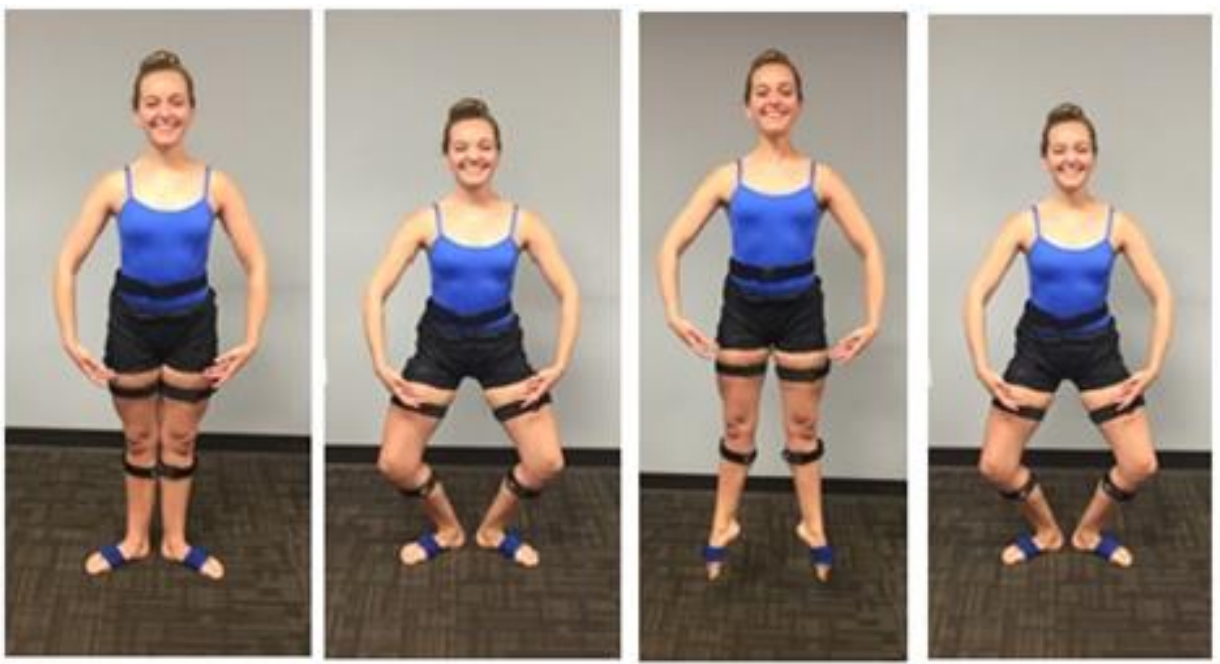

Figure 4. Left two frames: Preparation for a sauté in first position is in a plié. Middle: A sauté in mid-jump. Right: Landing of a sauté in first position is also in plié.

For the dependent variables of hip flexion, hip abduction, and hip rotation, side to side differences were compared via a mixed ANOVA. Each test ran side as an independent variable at two levels (right side and left side), and trial (jump 1, jump 8, and jump 15). As in the previous tests, the numbers were taken at peak knee flexion of each jump.

\section{RESULTS}

Statistically significant differences were found in right versus left hip abduction $(p=0.000)$. These average results are detailed further in Table 2 and Figure 5, which demonstrates that the average hip abduction for all jumps was 17.20 degrees of abduction on the left hip and 6.52 degrees of abduction on the right hip. This difference is 10.68 degrees from side to side such that the left hip was in 10.68 degrees more hip abduction on average during jumps than the right hip was. It should be noted that these numbers are all for hip abduction and that the dancers did not demonstrate average values of hip adduction for jumps.

Table 2. Means and standard deviations on right and left sides. All numbers were recorded in degrees. These are the means for all recorded jumps (1, 8, and 15). Numbers marked with "*" are statistically significant. Numbers marked with a "**" are clinically relevant

\begin{tabular}{|l|l|l|l|l|l|}
\hline & Right Mean & $\begin{array}{l}\text { Right Standard } \\
\text { Deviation }\end{array}$ & Left Mean & $\begin{array}{l}\text { Left Standard } \\
\text { Deviation }\end{array}$ & $\begin{array}{l}\text { Difference } \\
\text { Between Right } \\
\text { and Left Means }\end{array}$ \\
\hline Hip Abduction & $6.52^{*}$ & 8.50 & $17.20^{*}$ & 9.19 & $10.68^{\star *}$ \\
\hline Hip Flexion & 28.86 & 9.23 & 26.33 & 9.23 & 2.53 \\
\hline $\begin{array}{l}\text { Hip External } \\
\text { Rotation }\end{array}$ & 32.06 & 7.41 & 30.75 & 6.30 & 1.31 \\
\hline
\end{tabular}

Statistical significance was also found with hip flexion between jumps 1 and $8(p=0.000)$ and between jumps 1 and $15(p=0.006)$. This means that dancers had different hip flexion angles between the aforementioned jumps, meaning that the joint was not being loaded consistently each time. There was not a statistically significant difference in hip flexion between jumps 8 and $15(p=0.245)$.

There was no interaction effect of hip abduction between jump number and side $(p=0.470)$ or of hip external rotation between jump number and side $(p=0.599)$. No significant difference was found in: hip abduction between jumps $(p=0.062)$, hip flexion from side to side $(p=0.190)$, hip flexion from side to side between jumps $(p=0.972)$, hip external rotation between jumps $(p=0.366)$, or side to side hip external rotation $(p=0.562)$. Sphericity was assumed per Mauchly's Test of Sphericity in all conditions.

Of note, for the side-to-side differences, 19 participants were tested for jumps 1 and 8 . Due to one participant only performing 15 jumps as opposed to 16, only 18 participants were tested for jump 15. The researchers feel that completing all 16 jumps was important as the number of jumps is a large consideration in this study and deleting one subject on one jump should not affect the large levels of significance for hip abduction, nor would it change the hip flexion or external rotation significance. 


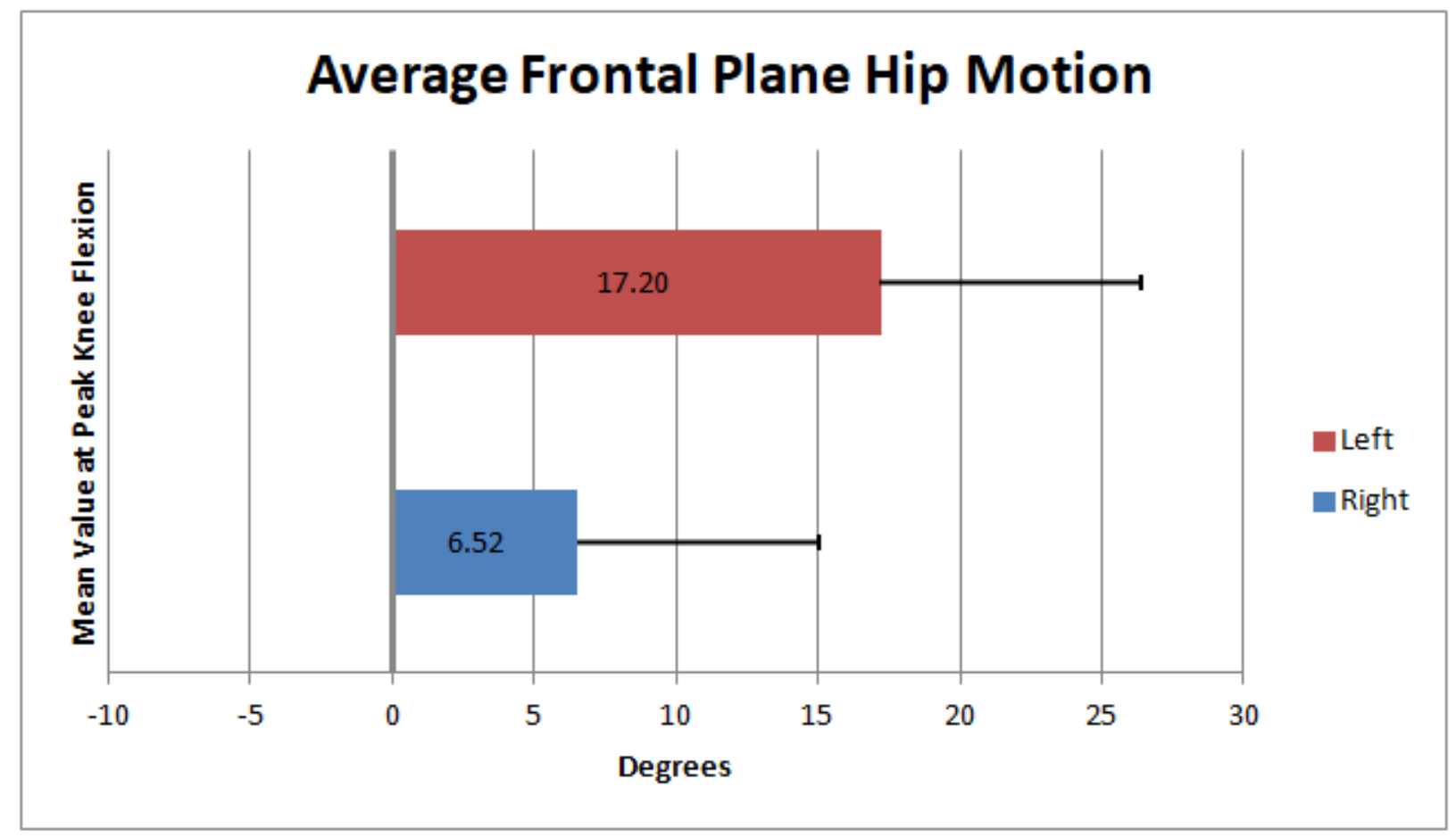

Figure 5. Frontal plane hip motion averages on the right versus left side with standard deviations included. Positive numbers indicate hip abduction, while negative numbers indicate hip adduction. There is a clinically relevant difference between right and left sides in hip abduction of $10.60^{\circ}$.

\section{DISCUSSION}

Two null hypotheses were able to be rejected: side to side differences in hip abduction and difference in hip flexion between jumps. The difference in hip abduction was both clinically and statistically significant $(p=0.000)$, with dancers achieving an average of 10.68 degrees more hip abduction on the left than the right. Though the average degrees are numerically considered clinically significant, the present study has done no investigation into whether this difference has a causal relationship to injury risk in dancers, and thus, no relationship of this manner can be assumed for this or any of the other variables presented. However, these are important values which help inform us that the joints are being loaded differently on each side, especially with hip abduction during jumps. Though the side to side difference in hip abduction did not change between jumps 1,8 , and 15 , it was present for all jumps and thus has the potential to worsen over time. If the dancers had been fatigued prior to testing, then perhaps additional side to side differences or pathomechanics would have been found. In a previous study, Cortes and colleagues tested female collegiate athletes both pre and post fatigue and found biomechanical differences in the hip flexion angle, knee flexion angle, and knee internal rotation angle. ${ }^{13}$ Dancers should be tested with similar strategies to examine whether these mechanical differences improve, worsen, or stay the same as what was found in this study.

The null hypothesis was also rejected for differences in hip flexion between jumps such that dancers had differing amounts of hip flexion between jumps 1 and $8(p=0.000)$ and 1 and $15(p=0.006)$. Though there was no side to side difference during these jumps, it still demonstrates that dancers have differing amounts of hip flexion during different jumps. This is especially important because there was a consistent tempo and all jumps were in the same position. If dancers' form changes even within these standardized conditions, it may break down even further when varying tempo (for instance, with performance music) or varying jumps (as in a variation). It is also worth noting that there was no significant difference in hip flexion between jumps 8 and $15(p=0.245)$, suggesting that dancers may have different form only for beginning jumps in a sequence and may settle into a more consistent form as they repeat jumps.

\section{Limitations}

This study had a small sample size and was restricted to first and second company members at one company. It may be beneficial to do a study with a variety of companies to account for different training styles. The authors also did not consider any previous injury or current pain that the dancers had, which could influence their performance on the task at hand. The participants were also 
allowed to warm up however they wanted, so they may not have been as fatigued as they would normally be by the time they perform jumps in class. Future studies to establish fatigue would benefit from having a uniform warm-up protocol that simulates what dancers would do before jumping in class or performances. Finally, this study only addressed jumps in first position; future studies should address jumps in various positions to establish when and in what positions form begins to break down.

\section{CONCLUSION}

Though the null was not able to be rejected for any of the other data that were run, this study has successfully established that dancers at a professional level are able to maintain consistent form for the first 15 jumps of a standard class, except for hip flexion and side to side hip abduction as discussed. It has also established that there are some significant side to side differences in dancers when jumping, though it is unclear as of yet whether symmetry is required in ballet or whether this is a learned and manageable effect of training throughout the years.

Future studies should assess dancers' form during jumps both before and after being fatigued, which can be assessed at least by heart rate. This would be beneficial to observe other potential pathomechanics which could be contributing to lower extremity injuries during jumps. Future studies done on single limb landing would also be useful to determine limb dominance for landing. A single limb study could further exemplify side to side differences, and could also be done both pre and post fatigue.

\section{REFERENCES}

1. Gamboa J, Roberts LA, Maring J, Fergus A. Injury patterns in elite preprofessional ballet dancers and the utility of screening programs to identify risk characteristics. Journal of Orthopaedic \& Sports Physical Therapy. 2008; 38(3): 126-136.

2. Smith T, Davies L, de Medici A, Hakim A, Haddad F, Macgregor A. Prevalence and profile of musculoskeletal injuries in ballet dancers: A systematic review and meta-analysis. Physical Therapy in Sport. 2015; 19: 50-56. Available from: Elsevier. Accessed 7 May 2020.

3. Allen N, Nevill A, Brooks J, Koutedakis Y, Wyon M. Ballet injuries: Injury incidence and severity over 1 year. Journal of Orthopaedic \& Sports Physical Therapy. 2012; 42(9): 781-790.

4. Ramkumar PN, Farber J, Arnouk J, Varner KE, McCulloch PC. Injuries in a professional ballet dance company: a 10year retrospective study. Journal of Dance Medicine \& Science. 2016; 20(1): 30. doi: http://dx.doi.org.bunchproxy.idm.oclc.org/10.12678/1089-313X.20.1.30

5. Picon AP, Rodes CH, Bittar A, Cantergi D, Loss J, Sacco ICN. Sauté external rotation in beginner and advanced ballet dancers trained in different background the turnout paradigm. Journal of Dance Medicine \& Science. 2018; 22(4): 1-6.

6. Harwood A, Campbell A, Hendry D, Ng L, Wild CY. Differences in lower limb biomechanics between ballet dancers and non-dancers during functional landing tasks. Physical Therapy in Sport. 2018; 32: 180-186. doi: 10.1016/j.ptsp.2018.05.005

7. Orishimo KF, Liederbach M, Kremenic IJ, Hagins M, Pappas E. Comparison of landing biomechanics between male and female dancers and athletes, part 1: Influence of sex on risk of anterior cruciate ligament injury. The American Journal of Sports Medicine. 2014; 42(5): 1082-1088. doi: 10.1177/0363546514523928

8. Ward R, Fong Yan A, Orishimo KF, Kremenic IJ, Hagins M, Liederbach M, Hiller CE, Pappas E. Comparison of lower limb stiffness between male and female dancers and athletes during drop jump landings. Scandinavian Journal of Medicine \& Science in Sports. 2018; 29(1). https://doi-org.bunchproxy.idm.oclc.org/10.1111/sms.13309

9. Ballet dictionary. American Ballet Theatre Web site. https://www.abt.org/explore/learn/ballet-dictionary/ Updated 2020. Accessed May 10, 2020.

10. Coplan JA. Ballet dancer's turnout and its relationship to self-reported injury. Journal of Orthopaedic \& Sports Physical Therapy. 2002; 32(11): 579-584.

11. Mangine GT, Hoffman JR, Gonzalez AM, et al. Bilateral differences in muscle architecture and increased rate of injury in national basketball association players. Journal of Athletic Training. 2014; 49(6): 794-799. doi: 10.4085/1062-605049.3.60

12. Balasubramanian $\mathrm{S}$, Abbas J. Comparison of angle measurements between Vicon and myoMOTIONTM systems. Noraxon web site. http://www.noraxon.com/wp-content/uploads/2016/05/myoMOTION-Validation-Study.pdf. Published August 23, 2013. Accessed June 10, 2020.

13. Cortes N, Quammen D, Lucci S, Greska E, Onate J. A functional agility short-term fatigue protocol changes lower extremity mechanics. J. Sports Sci. 2012; 30(8): 797-805. Published online 2012 Mar 19. doi: 10.1080/02640414.2012.671528 